\title{
An efficient interface between the solvers of Maxwell's equations and of the quasilinear kinetic equation for simulations of Ion Cyclotron Heating in Tokamak plasmas
}

\author{
M. Brambilla, R. Bilato \\ Max Planck Institute for Plasma Physics, EURATOM Association, Boltzmannstr. 2, \\ 85748 Garching, Germany
}

\begin{abstract}
Selfconsistent simulations of ion-cyclotron heating of tokamak plasmas require iterating between a solver of the wave equations in toroidal geometry and a solver of the Fokker-Planck equation describing the evolution of the ion distribution functions. A huge amount of information must be exchanged between the two codes at each iteration. For the package TORIC-SSFPQL we have developed an interface which substantially reduces the CPU and memory requirements for the storage and transmission of these data. We present this interface, and we take advantage of its efficiency to compare simulations in which the quasilinear diffusion operator in SSFPQL is evaluated superposing the TORIC results for all relevant toroidal modes excited by a given antenna with simulations using the fields of a single "representative" toroidal mode.
\end{abstract}

Keywords: full--wave equation, ion-cyclotron plasma heating, Fokker-Planck equation, quasilinear diffusion operator

\section{Introduction}

Auxiliary plasma heating with radio frequency (rf) waves in the ion cyclotron (IC) range of frequencies is used in several existing tokamaks and is planned for the next large fusion-oriented device, ITER [1]. Characteristic of IC heating is the production of energetic ions which can critically influence plasma stability and transport. In particular, fast ion populations influence the propagation and absorption of IC waves themselves. The interpretation 
of present-day IC heating experiments and the preparation of future ones, therefore, requires the simultaneous solution of Maxwell's equations to evaluate the fields and the power deposition in the plasma, and of the kinetic equation to determine the ion distribution functions. Each task represents a non-trivial numerical problem, which must be solved by a dedicated code; selfconsistent solutions, moreover, can only be obtained by iterating between the two codes. For this purpose, in the last few years several packages (CQL3DAORSA [2], GNET-TASK/WM [3], TORIC-SSFPQL [4, 5], ORBIT-AORSA [6]) have been developed in which a full-wave solver of the wave equations in toroidal geometry is coupled to a solver of the Fokker-Planck equation augmented by the quasilinear diffusion operator to describe the evolution of the ion distribution functions under the effect of resonant IC interactions.

Coupling two such codes presents some challenge. To achieve a satisfactory resolution both in real and in velocity space the amount of information to be exchanged between the two codes at each iteration is quite large, particularly from the kinetic solver to the solver of Maxwell's equations (the evaluation of the quasilinear operator in the kinetic code from the fields evaluated by the solver of Maxwell's equations also poses some non-trivial problems; they are, however, of a different nature, and will not be discussed in this note). To gauge the size of the task, we may note that the two codes must resolve variations on a rather different space scale. The radial mesh of the kinetic code must suffice to resolve the radial variation of the distribution functions and of the radial power deposition profiles. The radial mesh of the wave solver has to be much denser in order to resolve the shortest wavelength modes present in the plasma, namely ion Bernstein and/or ion cyclotron waves. The data from the kinetic code, therefore, must be sufficiently detailed to to allow a smooth interpolation to the denser mesh of the wave code. Smoothness is important, because the solution of wave problems is quite sensitive to irregularities of the coefficients of the wave equations, which are seen as unphysical scattering centers for short-wavelength modes. In simulations of medium-size tokamak plasmas this requirement can be satisfied by solving the kinetic equation on, say, 100 magnetic surfaces. With two ion species and a velocity space mesh of 250 points in modulus and 60 points in pitch-angle (the typical requirement for the accurate evaluation of the coefficients of the wave equations), the information to be transmitted to the wave code represents $\sim 510^{6}$ real numbers per iteration. For larger plasmas (JET, ITER) with several ion species, this amount increases by an 
order of magnitude or more.

Transmission of this information, moreover, is only part of the problem. The coefficients of the wave equations depend on the the generalized plasma dispersion functions (GPDFs), which are double integrals over the distribution functions with a resonant denominator (in a Maxwellian plasma the PDFs can be expressed in terms of the complex error function tabulated by Fried and Conte [7]; the definition for arbitrary distribution functions is given in the next section). In simulations of IC heating of medium-size tokamak plasmas a spectral code like TORIC makes several million calls to the routine which evaluates the GPDFs; for ITER, this number can exceed $10^{9}$, and increases linearly with the number of toroidal modes taken into account. Although a relatively fast and accurate method to evaluate the singular integrals is available [8]-[4], it would be hopelessly slow to perform the two velocity integrations at each call. A more reasonable approach might be to prepare a sufficiently dense table of values of the GPDFs, and let TORIC interpolate between these values as needed. Lookup tables, however, do not offer a fully satisfactory solution. To contain the amount of information mentioned above they would still have to be very extensive. Since, moreover, the meshes of the solver of Maxwell's equation are necessarily much finer than those of the solver of the kinetic equation, a large amount of interpolation in more than one variable (radial position and phase velocity) would be unavoidable. Even with this and similar short-cuts, therefore, the interface between the kinetic and the wave solvers could put a severe stress on the storage and CPU resources, and could still be the limiting factor to the efficiency of a complete code parallelization (at present, only TORIC has a parallelized version; the examples presented in this note, however, were executed with the serial version). It is, therefore, of some interest to make the interface between the two codes as efficient as possible. We describe here the interface developed for the package TORIC-SSFPQL.

To significantly reduce the load in the coupling between these two codes we have chosen to evaluate the GPDFs on each magnetic surface in SSFPQL as soon as the distribution functions have been obtained. The real and imaginary parts of the GPDF are then fitted with appropriate functions depending on a limited number of parameters, which are stored, and finally passed to TORIC as functions of the radial coordinate $\psi$. This has several advantages. Manifestly, it greatly reduces the amount of information to be temporarily 
stored by SSFPQL, written on an external file, and then read by TORIC. It also simplifies the task of transferring the information from one mesh to another, since each fitting parameter has to be separately interpolated in the radial variable only, while the GPDF itself should be simultaneously interpolated in the radial position and in phase velocity. We have also observed that on some platforms evaluating the GPDF using simple fitting functions it is faster than interpolating from tables. Depending on the size of the plasma, moreover, a run of TORIC using the information from SSFPQL for arbitrary distribution functions can be up to $20 \%$ faster than a run for a Maxwellian plasma in which the PDF is evaluated with the otherwise very fast (and, of course, much more accurate) Gautschi algorithm for the complex error function $[9]$.

The execution speed provided by this interface allows one to increase the accuracy of simulations of high-power heating experiments. Because of the heavy CPU and memory requirements and the long execution times, iterations between the Maxwell and kinetic solver are mostly made taking into account only a single "representative" toroidal Fourier mode of the wave field. While this is to some extent justified by the weak dependence of the quasilinear operator on the toroidal wavenumber, it is not entirely satisfactory, since IC antennas always excite a broad spectrum of toroidal modes. With the full-wave code EVE, moreover, Dumont [10] has recently performed extensive simulations of IC on ITER with complete spectral scans, although only with Maxwellian distribution functions, and pointed out that such scans are essential to compare the efficiency of different excitations of multi-strap antennas. We have exploited the execution speed of the TORIC-SSFPQL package to perform iterations in which the quasilinear diffusion coefficient in SSFPQL is evaluated superposing the results of TORIC for the full nominally antisymmetric spectrum of toroidal modes radiated by the two-strap IC antenna of ASDEX Upgrade, which covers the range of toroidal wavenumbers $-35 \leq n_{\varphi} \leq+35$. Some data on this simulation at the end of section 4 illustrate the efficiency of the interface.

The rest of this note is structured as follows. In section 2, the equations solved by TORIC and SSFPQL are briefly recalled, to better illustrate the problems presented by the interface between the two codes. The next two sections discuss our implementation of this interface, with particular attention to the choice of adequate fitting functions for the complex GPDFs. The results of a few self-consistent iterations taking into account the full spectrum of toroidal Fourier modes are presented in section 4, and compared with similar simula- 
tions using a single representative mode. In the last section we present some conclusions, and briefly mention the limitations which might be encountered to extend our approach to other packages.

\section{The codes TORIC and SSFPQL.}

The structure and the coefficients of the wave equations solved by TORIC can be found in [11]. To discuss the problems posed by the interface between the wave and the kinetic solver concretely, it will suffice here to recall that in toroidal geometry the relation between the rf current $\vec{J}$ and the wave field $\vec{E}$ in the plasma (the "constitutive relation") obtained from the linearized Vlasov equation is a non-local, integral functional of the distribution functions of ions and electrons. This relation is substantially simplified by introducing the spectral representation of the wave electric field

$$
\vec{E}=\sum_{m_{\vartheta}, n_{\varphi}} E^{\left(m_{\vartheta}, n_{\varphi}\right)}(\psi) e^{i\left(m_{\vartheta} \vartheta+n_{\varphi} \varphi-\omega t\right)}
$$

Here the radial coordinate $\psi$ is a label of magnetic surfaces, $\varphi$ is the geometrical toroidal angle, and $\vartheta$ a poloidal angle. Each Fourier component in Eq. (1) defines its own "parallel wavevector component"

$$
k_{\|}^{m_{\vartheta}, n_{\varphi}}(\psi, \vartheta)=\frac{m_{\vartheta}}{N_{\vartheta}} \cos \Theta+\frac{n_{\varphi}}{R} \sin \Theta
$$

where $R$ is the major radius, $\tan \Theta=B_{\text {pol }} / B_{\text {tor }}$ is the pitch of the confining magnetic field, and $N_{\vartheta}^{2}=g_{\vartheta \vartheta}$ is the element of the metrics of toroidal coordinates which reduces to the minor radius $r$ squared of the magnetic surface in the limit of circular poloidal cross-sections. Since $k_{\|}^{m_{\vartheta}, n_{\varphi}}$ depends on position only on the slow scale of the equilibrium, to a first approximation the plasma response to rf waves depends on $k_{\|}^{m_{\vartheta}, n_{\varphi}}$ as in the uniform plasma limit. That this response is different for each Fourier component of the field, on the other hand, is a consequence of toroidicity (we disregard here for simplicity other toroidicity-induced corrections to the plasma response due to the parallel acceleration of particles transiting through resonances, and which can be reduced to a modification to $k_{\|}^{m_{\vartheta}, n_{\varphi}}$ [12]; they are taken into account, however, in TORIC). For TORIC, this has the consequence of greatly increasing the number of times the GDPS which enters the coefficients of the wave equations, and describes the effects of parallel dispersion through its 
dependence on $k_{\|}^{\left(m_{\vartheta}, n_{\varphi}\right)}$.

The GPDF is defined for a real argument as

$$
\tilde{Z}_{i}^{(q)}(\psi, x)=\frac{1}{\sqrt{\pi}} \mathrm{P} \int_{-\infty}^{\infty} \frac{F_{i}^{(q)}(\psi, u)}{u-x} \mathrm{~d} u+i \sqrt{\pi} F_{i}^{(q)}(\psi, x), \quad q=0,2
$$

where the argument $x$ has the form $x=\left(\omega-p \Omega_{i}\right) / k_{\|}^{m_{\vartheta}, n_{\varphi}} v_{t h 1}$, with, in TORIC, $p=0$, 1, or 2 , and $k_{\|}^{m_{\vartheta}, n_{\varphi}}$ defined in Eq. (2) above. The singular integral in the real part (Hilbert transform) is intended as the Cauchy principal part. The functions $F_{i}^{(q)}(\psi, u)$ in the numerator of the real part and in the imaginary part are the two perpendicular moments of the quasilinear ion distribution function

$$
F_{i}^{(q)}(\psi, u)=\int_{0}^{+\infty} w^{q} F_{i}(\psi, u, w) w \mathrm{~d} w
$$

with $w=v_{\perp} / v_{t h i}, u=v_{\|} / v_{t h i}$, parallel and perpendicular referring to the direction of the static magnetic field. These moments and their Hilbert transform describe respectively wave dissipation and dispersion contributed by charged particles of species $\alpha$. In SSFPQL the singular integral is evaluated with a slightly simplified version of an algorithm first proposed by Valeo [13] (see also [8]). Examples of GPDFs for non-Maxwellian distribution functions can be seen in Fig. 3 below.

In the ion cyclotron range of frequencies the electron distribution remains always very close to Maxwellian, even in the presence of rf-driven current. The electron contributions (6), therefore, can be expressed in terms of the PDF tabulated by Fried and Conte [7], to which Eq. (3) reduces for Maxwellian distribution functions. In the presence of IC heating, by contrast, the distribution functions $F_{i}(\psi, u, w)$ of the ions differ substantially from Maxwellians, and must be evaluated by solving the quasilinear kinetic equation. In our package, this is the task of the SSFPQL code [4], which solves the steady-state surface-averaged linearized Fokker-Planck equation

$$
0=\left(\frac{\partial F_{i}}{\partial t}\right)_{\text {coll }}+\left(\frac{\partial F_{i}}{\partial t}\right)_{\mathrm{ql}}+\mathcal{S}_{i}^{\Delta \mathrm{T}}(\psi, v)+\mathcal{S}_{i}^{\mathrm{nbi}}(\psi, v, \mu)+\mathcal{L}_{i}^{\mathrm{fil}}(\psi, v, \mu)
$$

for ions heated at the fundamental and first harmonic of the IC resonance and/or by neutral beam injection (NBI) in tokamak plasmas, using the output of TORIC to build the QL diffusion coefficient (QLDC) on each magnetic 
surface. In Eq. (5) energy losses to the background plasma, described by the linearized collisional operator $\left(\partial F_{i} / \partial t\right)_{\text {coll }}$, balance the gains described by the quasilinear operator $\left(\partial F_{i} / \partial t\right)_{\mathrm{ql}}$ and by the NBI source $\mathcal{S}^{\text {nbi }}$. The isotropic source term $S^{\Delta \mathrm{T}}$ is added to allow steady-state solutions in which different background species are maintained at different temperatures in the absence of external heating, and the term $\mathcal{L}_{i}^{\text {fil }}$ describes losses of fast ions during thermalization. The explicit form of these terms can be found in [4]. Here it suffices to recall that in the quasilinear term

$$
\frac{1}{\nu_{\text {coll }}}\left(\frac{\partial F_{i}}{\partial t}\right)_{\mathrm{ql}}=\frac{1}{v_{\perp}} \frac{\partial}{\partial v_{\perp}}\left(v_{\perp} D_{\mathrm{ql}}^{p}\left(v_{\perp}\right) \frac{\partial F_{i}}{\partial v_{\perp}}\right)
$$

the QLDC for heating at the pth IC harmonic ( $p=1$, fundamenta; $p=2$, first harmonic) has the form

$$
D_{\mathrm{ql}}^{p}=D_{0}\left|E_{+} J_{p-1}\left(\xi_{\perp} w\right)+E_{-} J_{p+1}\left(\xi_{\perp} w\right)\right|^{2}
$$

where $E_{ \pm}$are the circularly polarized components of the wave electric field, and, in the Bessel functions, $\xi_{\perp}=k_{\perp} v_{t h i} / \Omega_{c i}$ and $w=v_{\perp} / v_{t h i}$. SSFPQL obtains the solution of Eq. (5) in the form of a truncated series in Legendre polynomials, $P_{n}(\mu)$, of the pitch angle $\mu=v_{\|} / v$,

$$
F(\psi, v, \mu)=\sum_{n} F_{n}(\psi, v) \mathrm{P}_{n}(\mu)
$$

A special Bessel function identity (eqn (16) of [4] and recently further discussed in [14]) has been used to obtain a convergent Legendre series representation of $D_{\mathrm{ql}}^{p}$. When the rf power is high, and particularly in the presence of simultaneous NBI, a large number (up to 60) terms might have to be retained in Eq. (6) to guarantee convergence of the distribution functions in a sufficiently large velocity domain.

In writing Eqs. (6) and (7) we have ignored the effects of toroidal trapping on the ion QL diffusion coefficient. We have recently extended SSFPQL to take this effect into account, but the more complicated expression for $D_{\mathrm{ql}}^{p}$ in the presence of toroidal trapping does not change anything to the interface between the two codes. 


\section{Building the interface.}

As mentioned in the introduction, the structure of the problem makes it necessary to iterate between TORIC and SSFPQL to obtain a consistent solution. In the first run TORIC assumes Maxwellian distribution functions. At each iteration, SSFPQL uses the fields evaluated by the previous run of TORIC to build the QL diffusion coefficient $D_{\mathrm{ql}}^{p}$, solves the kinetic equation at each radial point, and reevaluates the GPDFs using the resulting distribution functions. All the information needed by TORIC is contained in these functions, which from the present point of view depend only on the ion species and the radial position $\psi$. In turn, TORIC solves again the wave equations again using the updated GPDFs in the coefficients of the wave equations. For this purpose, it must import the necessary information on the GPDFs from SSFPQL: for the reasons mentioned in the introduction, we have discarded the more cumbersome alternative of importing instead information on the distribution functions and constructing the GPDF in TORIC itself. Achievement of consistency is checked by comparing the power deposition profiles predicted by the two codes. In TORIC they are evaluated in terms of the antihermitian part of the coefficients of the wave equations, while SSFPQL evaluates them as moments of the distribution functions weighted with $D_{\mathrm{ql}}^{p}$. The iteration is stopped when the rf power deposited in each ion species evaluated by the two codes differs by less than a specified amount, typically one or two per cent, at all points of the radial mesh of SSFPQL (in the first iteration the predictions of the two codes might differ by a factor 2 or more). The number of iterations needed to reach such an agreement varies from 4 or 5 at low power density (everywhere less than $1 \mathrm{~W} / \mathrm{cm}^{3}$ ) to 15 or more at high power (with peaks of several $\mathrm{W} / \mathrm{cm}^{3}$ ). The relatively large number of iterations needed at high power was the incentive to make the interface as efficient as possible.

Because of surface averaging, the radial coordinate $\psi$ of the tokamak configuration enters the kinetic equation only as a parameter, so that SSFPQL solves Eq. (5) separately on each magnetic surface. This makes it convenient to evaluate the GPDFs at each point of the radial mesh of SSFPQL as soon as the solution of the kinetic equation is available, thereby eliminating the need for storing the entire distribution functions. As mentioned in the introduction, we have chosen to fit the GPDFs with functions depending on a limited number of parameters. To store and transmit only these parameters reduces substantially the size of the storage, and greatly simplifies in TORIC the task 
of evaluating the GPDF on its dense radial mesh and for a huge number of values of the phase velocity argument. We describe next how the fitting has been implemented.

Beginning with the moments (4), we recall that they enter in the imaginary part of the GPDF, and give the number of resonant particles with normalized parallel velocity $u=\left(\omega-p \Omega_{c i}\right) / k_{\|}^{\left(m_{\vartheta}, n_{\varphi}\right)} v_{t h i}$ which contribute to damping of the waves. For large $|u|$ this number decreases exponentially, although in the presence of IC heating the decrease can be substantially slower than in the case of a Maxwellian plasma. Thus the moments are needed by TORIC only in a finite range, say $|u| \lesssim U$, with, typically, $U \sim 7$ to 12 (corresponding to energies 50 to 150 times the background temperature) chosen so that the contribution to wave damping by ions outside this range is negligible. The other bit of information needed to devise an efficient fitting is the behavior of $F_{i}^{(q)}(u)$ for low velocities, $|u| \ll 1$. From the properties of the collision operator, one can show that the two-dimensional distribution function $F_{i}(v, \mu)$ always has its maximum at the origin; in the presence of strong directed NBI, however, the moments $F_{i}^{(q)}(u)$, particularly the second $(p=1)$ which is more heavily weighted for high perpendicular velocities, can be peaked slightly away from $u=0$. The accurate determination of the position $u_{M}$ where $F^{(q)}(u)$ is stationary turns out to be critical for the success of the fitting.

For the purpose of fitting, we define functions $\lambda_{q}(u)$ related to the moments by

$$
F^{(q)}(u)=\exp \left[A_{q}-\left(u-u_{M}\right)^{2} \lambda_{q}(u)\right] \quad q=0,2
$$

where $u_{M}$ is the stationary point of $F^{(q)}(u)$ near or at the origin (velocities are normalized to the local thermal speed). Thus, by definition,

$$
A_{q}=\log \left[F^{(q)}\left(u_{M}\right)\right]
$$

and

$$
\lambda_{q}(u)=\frac{\log \left[F^{(q)}\left(u_{M}\right)\right]-\log \left[F^{(q)}(u)\right]}{\left(u-u_{M}\right)^{2}}
$$

Fitting $\lambda_{q}(u)$ is much easier, and it produces more accurate results than fitting $F^{(q)}(u)$ itself. Note, in particular, that for unperturbed Maxwellians 
$\lambda(u) \equiv 1$ identically. In general, $\lambda_{q}(u)$ is formally indeterminate for $u \rightarrow u_{M}$; however, we have

$$
\lambda_{q}\left(u_{M}\right)=-\lim _{u \rightarrow u_{M}} \frac{F^{(q)^{\prime}}(u)}{2 F^{(q)}(u)\left(u-u_{M}\right)}=-\frac{F^{(q)^{\prime \prime}}\left(u_{M}\right)}{2 F^{(q)}\left(u_{M}\right)}
$$

where primes denote derivation with respect to $u$.

The functions $\lambda_{q}(u)$ are fitted (separately in $u_{M} \leq u<U$ and in $-U \leq$ $u \leq u_{M}$ if the symmetry of the distribution function cannot be assumed) with a polynomial

$$
\lambda_{q}(u) \simeq y_{q}(u)=b_{0}^{(q)}+b_{1}^{(q)} \zeta+b_{2}^{(q)} \zeta^{2}+\cdots+b_{n}^{(q)} \zeta^{n} \quad \zeta=u-u_{M}
$$

whose coefficients are evaluated by Lagrangian interpolation based on the zeros of the Chebyshev polynomial of order $N+1$ mapped on the interval $u_{M} \leq u<U$ or $-U \leq u \leq u_{M}$. To test the quality of the fitting obtained, in addition to monitoring the largest difference between $\lambda_{q}(u)$ and $y_{q}$, we compare the integrals

$$
\int_{-\infty}^{+\infty} F_{i}^{(0)}(u) \mathrm{d} u=1 \quad \quad \int_{-\infty}^{+\infty} F_{i}^{(2)}(u) \mathrm{d} u=\frac{E_{\perp}}{T_{i}}
$$

which are a direct measure of IC damping by ion species $i$. With polynomials of order 10 the relative difference between the integrals evaluated with the exact and the fitted distribution is typically $\ll 10^{-3}$. In our tests, failure of the fitting occurred only when the number of Legendre polynomials used in SSFPQL to represent the distribution function was too small to ensure convergence of the representation (14) in a sufficiently large velocity domain. When this happens, SSFPQL prints a warning and stops.

To fit the real part of the GPDF, $\operatorname{Re}\left[\tilde{Z}^{(q)}(x)\right]$, the general behaviour of this function suggests instead to use rational functions. In the Maxwellian limit this function is antisymmetric in $x$, linear in $x$ near the origin, and decreases as $x^{-1}$ for large argument. The fitting function, therefore, has been chosen of the form

$$
\begin{aligned}
& \operatorname{Re}\left[\tilde{Z}^{(q)}(x)\right] \simeq z_{q}(\zeta)=\frac{\zeta P_{q}(\zeta)}{1+\zeta^{2} Q_{q}(\zeta)}=\frac{\zeta\left(p_{0}^{(q)}+p_{1}^{(q)} \zeta+\cdots+p_{n}^{(q)} \zeta^{n}\right)}{1+\zeta^{2}\left(q_{0}^{(q)}+q_{1}^{(q)} \zeta+\cdots+q_{n}^{(q)} \zeta^{k}\right)} \\
& \zeta=x-x_{Z}
\end{aligned}
$$


Here, again, the 'shift' $x_{Z}$ is introduced to take into account the possible asymmetry of the moments of the distribution function; it is usually smaller than $u_{M}$ in Eq. (13), and is estimated from the derivative of $F^{(q)}(u)$ at the origin. To initialize the fit, the polynomial $Q_{q}$ in the denominator is assumed to be unity, and the coefficients of $P_{q}$ in the numerator are evaluated imposing

$$
\operatorname{Re}\left[\tilde{Z}^{(q)}\left(x_{j}\right)\right]=\frac{\zeta_{j} P_{q}\left(\zeta_{j}\right)}{1+\zeta_{j}^{2}} \quad j=1,2, \ldots, N
$$

at $n$ equidistant points in the interval $u_{Z} \leq x \leq U$ or $-U \leq x \leq u_{Z}$ (positive and negative velocities are again treated separately, unless the distribution function can be assumed to be symmetric in the parallel velocity). The coefficients of the polynomial $Q_{q}$ are then obtained with a standard fitting procedure, without changing $P_{q}$. Again, we have never observed the occurrence of a fitting failure (e.g. a pole in the denominator in the range of fitting) when the Legendre representation of the solutions of the kinetic equations is properly convergent. Nevertheless, for safety, SSFPQL will stop if the largest relative difference between the exact and the fitting function cannot be reduced below $2 \%$.

In contrast to the imaginary part, $\operatorname{Re}\left[\tilde{Z}^{(q)}(x)\right]$ is needed also outside the fitting range $|x| \leq U$. For large argument it is best evaluated from the asymptotic development

$$
\begin{aligned}
\tilde{Z}_{i}^{(q)}(\psi, x) & \simeq-\frac{1}{x} \sum_{k} \frac{J_{k}^{(q)}}{x^{k}} \\
J_{k}^{(q)} & =\frac{1}{\sqrt{\pi}} \int_{-\infty}^{\infty} F_{i}^{(q)}(\psi, u) u^{k} \mathrm{~d} u
\end{aligned}
$$

For this purpose the first ten parallel moments (more than actually ever needed) of $F_{i}^{(q)}$ are also evaluated and passed to TORIC. The odd ones are always much smaller than the even ones, and they vanish if the distribution functions are symmetric.

\section{An example.}

To illustrate the performances of the interface described in the previous section we discuss two simulations of a typical IC minority heating experiment with the TORIC-SSFPQL package. We have used the parameters of the 

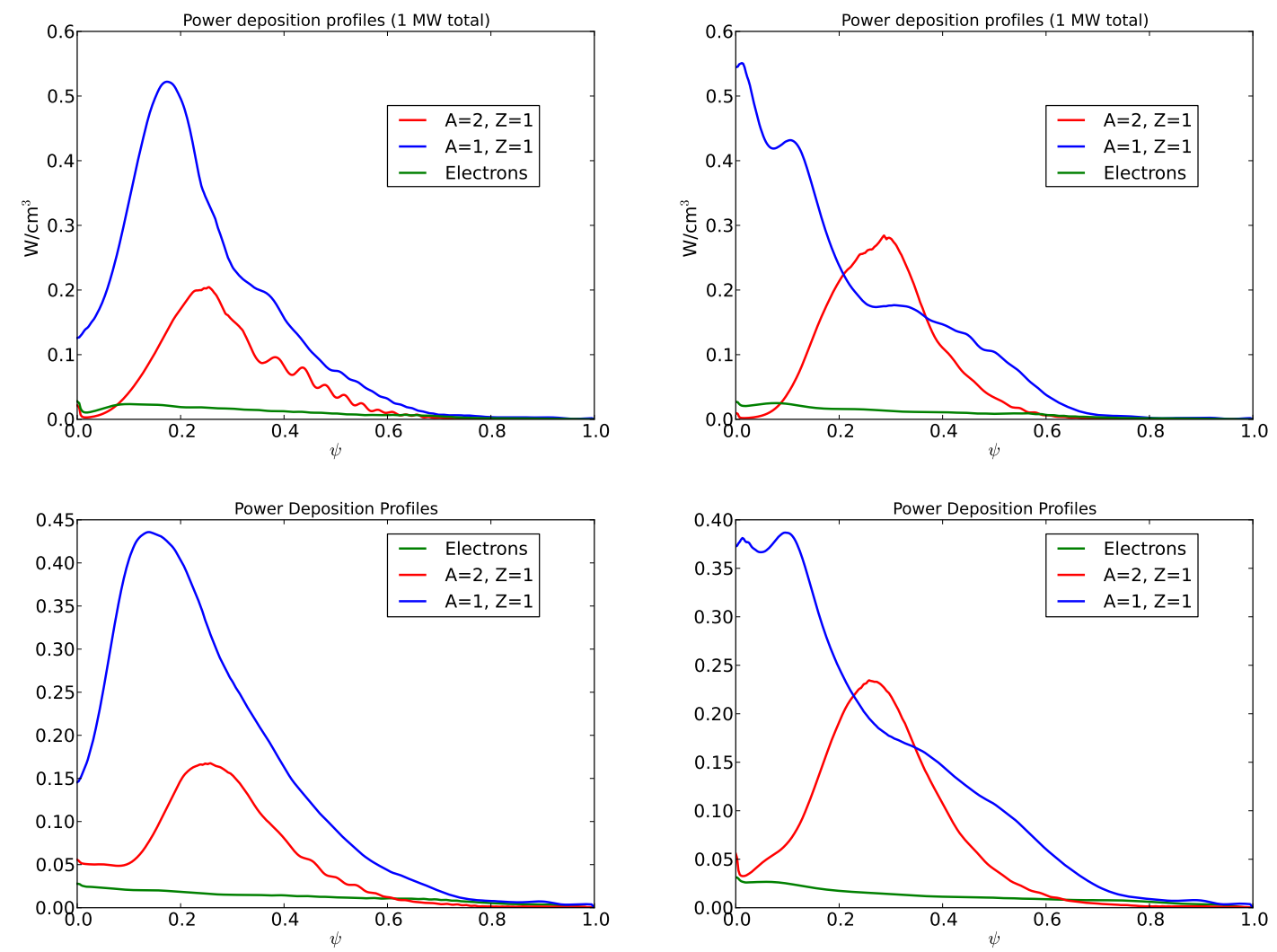

Figure 1: Power deposition profiles (W/ $\mathrm{cm}^{3}$ per MW of total power coupled). Above left: $n_{\varphi}=12$, Maxwellian distribution functions; above right: $n_{\varphi}=12$, selfconsistent quasilinear distribution functions. Below left: full toroidal spectrum, Maxwellian distribution functions; below right: full toroidal spectrum, selfconsistent quasilinear distribution functions. $A$ and $Z$ are the atomic mass and charge of the two ion species. 

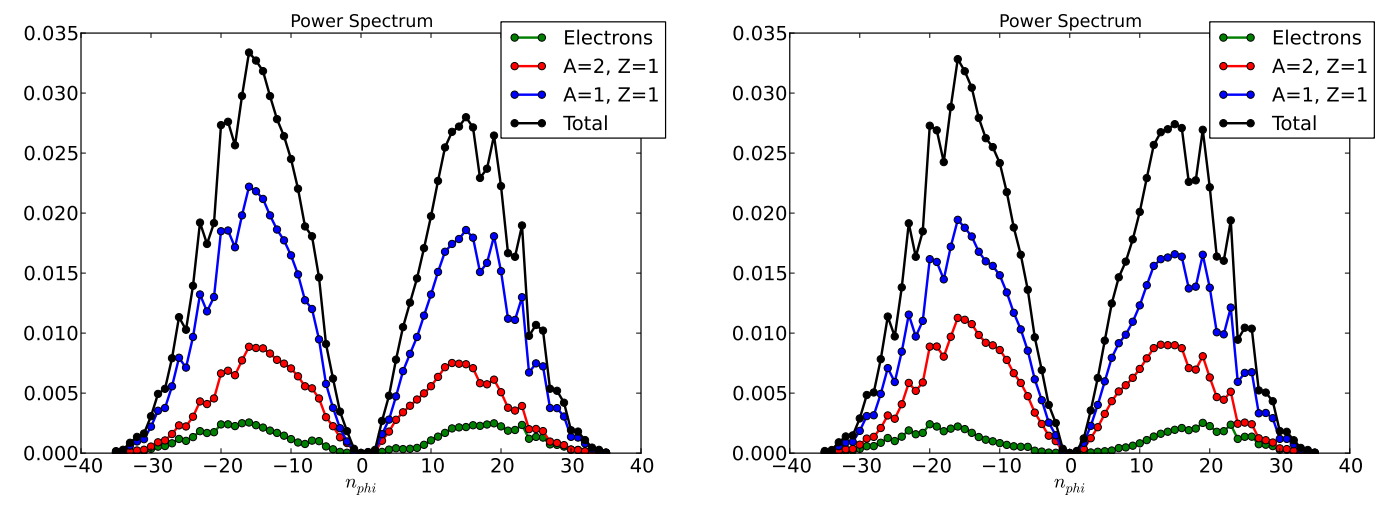

Figure 2: Repartition of the launched power between toroidal modes. Left: Maxwellian plasma, right: selfconsistent quasilinear distribution functions.

ASDEX Upgrade [15] tokamak (major radius $1.7 \mathrm{~m}$, horizontal minor radius $0.465 \mathrm{~m}$, ellipticity 1.51 , central density $n_{e}(0)=7.110^{19} \mathrm{~m}^{-3}$, central temperature $T_{i}=T_{e}=1.88 \mathrm{keV}, 5 \%$ hydrogen in deuterium). With a central magnetic field of $1.99 \mathrm{~T}$ and a frequency of $30.5 \mathrm{MHz}$ the fundamental IC resonance of hydrogen and the first harmonic of deuterium where located $0.055 \mathrm{~m}$ to the inside of the magnetic axis. The total $\mathrm{rf}$ power was assumed to be $4 \mathrm{MW}$. Note that in ICRF experiments in a medium-size tokamak the rf power density is much higher than in large devices such as JET or ITER, and their simulation, therefore, poses more severe challenges on the convergence of the consistency iteration between the two codes.

Figure 1 shows the power deposition profiles obtained under four different assumptions. The first two plots are the results of TORIC for a single 'representative' toroidal mode $\left(n_{\varphi}=12\right.$ in this case). Below are the results of a simulation in which TORIC was made to scan the full toroidal spectrum excited by the ASDEX Upgrade IC antenna (two straps excited antisymmetrically). To the left are the initial profiles evaluated for Maxwellian distribution functions, to the right those for the selfconsistent quasilinear distributions. The toroidal power spectrum predicted assuming Maxwellian and selfconsistent distribution functions are shown in Fig. 2. The differences are not large; a close look reveals mainly a moderate shift of the power deposition in favor of absorption by deuterium. 
We may note that $D_{\mathrm{ql}}^{p}$ is the sum of independent contributions from each toroidal mode of the fields. The dependence of the coefficients of the wave equations on the distribution functions, however, indirectly introduces a nonlinear coupling between these modes. This does not alter in any way the structure of the iteration, but it might be expected to have a non-negligible influence on the results. Nevertheless, neither the power absorption profiles nor the global power repartition between species (table 1) are substantially different between the two simulations. With the full toroidal spectrum the profiles are somewhat broader and smoother, and the peak value of the power deposition correspondingly slightly lower. The contributions of partial waves with large values of $k_{\|}$favors direct deposition in the hydrogen and in the electrons at the expense of deuterium; In this case, however, this is more than compensated by enhanced absorption by deuterium of toroidal modes with moderate and small toroidal wavenumbers. After collisional repartition (values in parentheses), the power deposited in the electrons is further somewhat increased because of the slightly higher energy content in the hydrogen suprathermal tail.

These effects, however, are relatively small: it is clearly more important to iterate between the two codes until both calculate the absorption with the same distribution function than to perform every time a full scan over toroidal modes. Indeed, at least in the case of antisymmetric spectra, restricting the simulation to a single 'representative' toroidal mode is not expected to introduce major inaccuracies, since the quasilinear operator for IC heating by the compressional wave depends only weakly on the value of $k_{\|}$. Nevertheless, it can be useful to have the possibility to confirm this expectation with a simulation lasting only a few hours when executed on an Intel i7-2670QM (2.2 MHz) CPU.

\begin{tabular}{|l|c|c|c|}
\hline & $\begin{array}{c}\text { hydrogen } \\
{[\%]}\end{array}$ & $\begin{array}{c}\text { deuterium } \\
{[\%]}\end{array}$ & $\begin{array}{c}\text { electrons } \\
{[\%]}\end{array}$ \\
\hline$n_{\varphi}=12$ Maxw. & 65.22 & 28.58 & 6.20 \\
\hline$n_{\varphi}=12$ Qlin. & $55.78(1.99)$ & $37.64(48.27)$ & $5.58(49.74)$ \\
\hline full scan, Maxw. & 66.70 & 25.71 & 7.59 \\
\hline full scan, Qlin. & $59.29(2.05)$ & $33.55(42.54)$ & $7.16(55.41)$ \\
\hline
\end{tabular}

Table 1: The rf power deposited in each species (in parentheses, the values after collisional redistribution). 
We next present a few examples of the fitting procedure, taken from the final iteration of the full spectrum simulation. In this iteration TORIC and SSFPQL use the same distribution functions, and predict the same deposition profiles to an accuracy of $\pm 2 \%$. In figure 3 are plotted the GPDFs relevant for this IC scenario, namely $\tilde{Z}^{(2)}(z)$ for the majority, and $\tilde{Z}^{(0)}(z)$ for the minority. They are shown here at the point of peak absorption for each species, where the deviations from the Maxwellian limit, shown for comparison with dashed lines, are largest. We may note that in the case of fundamental heating the area under the plot of $\operatorname{Im} Z^{(0)}(z)$, which is a measure of fundamental IC absorption, is always unity (see Eq. (14)): the main effect of suprathermal minority ions is a marked Doppler broadening of the absorption layer, as visible in the power deposition profiles. In the case of harmonic heating, by contrast, this area is proportional to the perpendicular energy content of the distribution function, in agreement with the fact that harmonic IC heating is a finite Larmor radius effect. Each modification of the GPDF, taken alone, would increase damping by the corresponding ion species; the outcome of the competition between the two species depends on the local temperature, the minority concentration, and the strength of the single transit absorption, and it is quite difficult to predict in advance. It is, therefore important that the fitting of $\operatorname{Im}\left(\tilde{Z}^{(q)}\right)$ should be as accurate as possible. The real part of $Z^{(q)}$ plays a less critical role: the only appreciable effect of the increase in modulus of $\operatorname{Re}\left(\tilde{Z}^{(2)}\right)$ near $\omega=2 \Omega_{c D}$ is that ion Bernstein waves excited by mode conversion at the nearby ion-ion resonance have a somewhat longer wavelength than in the Maxwellian limit. This ensures that numerical resolution requirements on the mesh of TORIC do not become more severe in the presence of suprathermal ions.

Figures 4 and 5 show the results of fitting the real and imaginary part of the GPDFs of figures 3. Although they refer to the point of maximum absorption, where the demands on the fitting procedure are most severe, the difference between the true functions (blue curves) and their approximations by rational functions (red curves) are quite small. In the exponent $\lambda_{0}(u)$ for the hydrogen, one discerns small discontinuities due to the fact that to evaluate the moment $F_{\mathrm{H}}^{(0)}(u)$ one has to integrate the distribution function along straight lines $\left(v_{\|}=\right.$constant $)$in a domain defined by an inequality of the form $F_{\mathrm{H}}(v, \mu)>\epsilon, \epsilon \ll 1$ (cfr. Eq. 8), whose contour is roughly a very elongated ellipse, and is located in a domain in which the derivatives of $F_{\mathrm{H}}(v, \mu)$ are exponentially small. These discontinuities can hardly be seen 

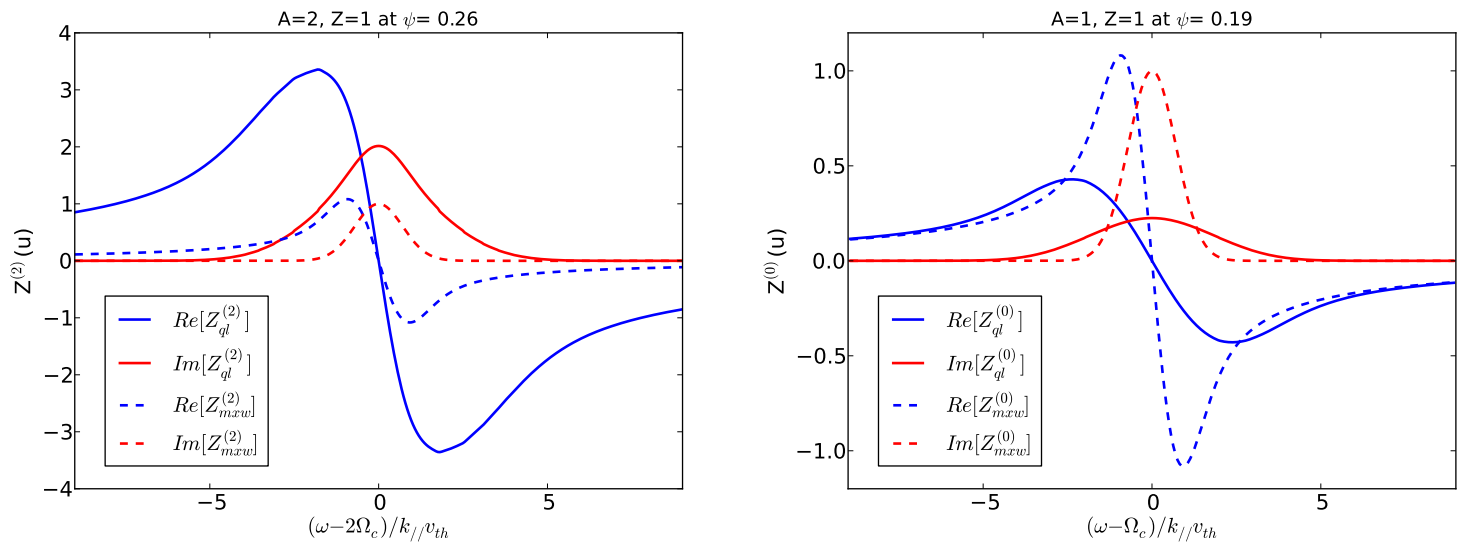

Figure 3: The generalized plasma dispersion functions at the radial point of maximum absorption. Left: deuterium, first harmonic (at $\psi=0.26$ ); right: hydrogen, fundamental (at $\psi=0.19$ ). The dashed curves are the same PDFs for the Maxwellian plasma.

in the plot of $\operatorname{Im}\left(Z^{(0)}(x)\right)=\sqrt{\pi} F_{\mathrm{H}}^{(0)}(x)$ (full red curve in figure (3), left), but are amplified by the definition of $\lambda_{0}(u)$. They are nicely smoothed out by the fitting function.

Some data from the run from which the results just presented have been taken can illustrate the efficiency of our approach. The simulation has converged in nine iterations, and was performed in 3h 37' on an Intel i7-2670QM $(2.2 \mathrm{MHz}) \mathrm{CPU}$. It is interesting to have a look to the repartition of the CPU time between the different tasks. Of the total time, TORIC used 3h 02', and SSFPQL 33'. In TORIC, the largest overheads were the evaluation of large Larmor radius corrections to the coefficients of the wave equations describing propagation and damping of ion Bernstein wave [16] with 51', and, after the inversion of the stiffness matrix, the evaluation of the power deposition profiles with 33'. The GPDF was evaluated, using the fitting coefficients transmitted by SSFPQL, 1,417,449,663 times in 3' 14", while the plasma dispersion function of the Maxwellian plasma (for the electrons, and for the ions in the first iteration and later in the plasma periphery where deviations from Maxwellian were insignificant) 861,446,209 times in 4' 16". In SSFPQL the evaluation of the moments of the distribution function (eq. 4 below) was performed 2020 times in 41", the singular integrals in the definition of the generalized Plasma Dispersion Function 4040 times in 0.98", and the deter- 

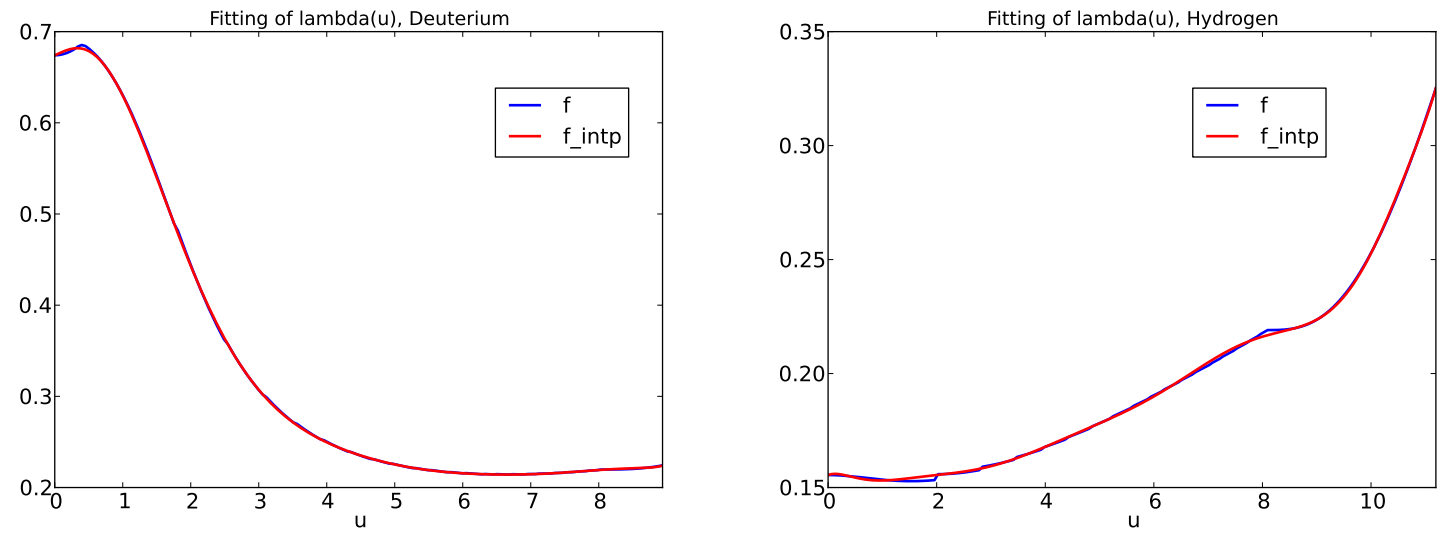

Figure 4: Fitting the exponent $\lambda(u)$ in the imaginary part of the GPDFs. Left: deuterium; right: hydrogen.
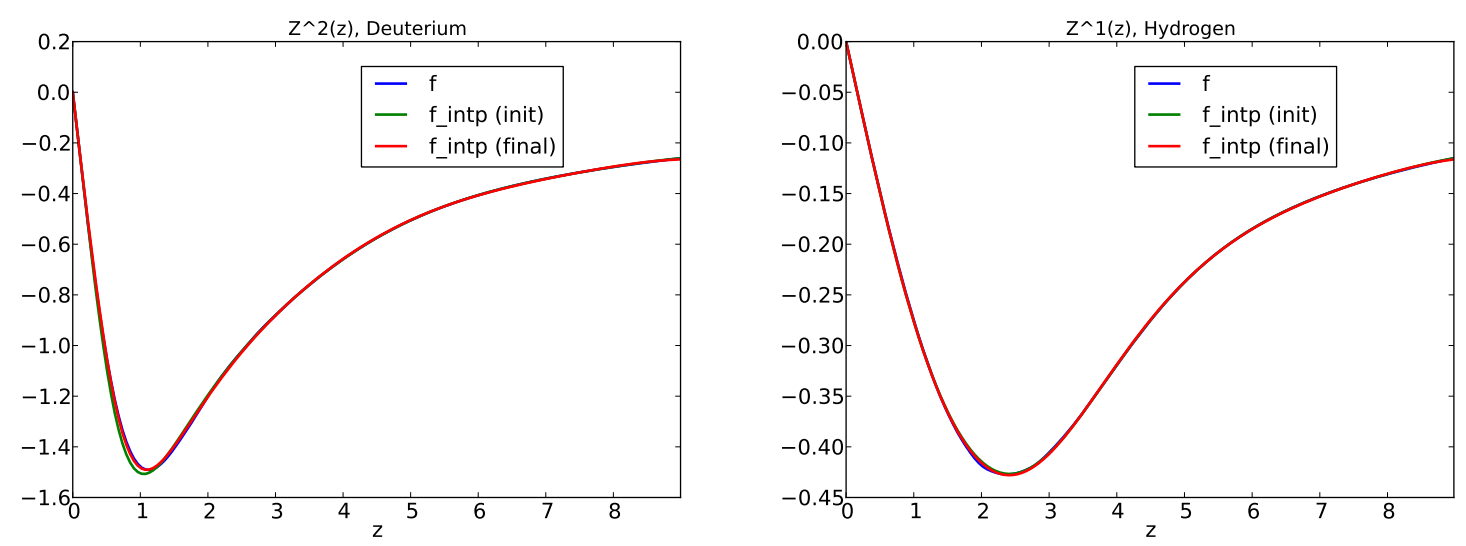

Figure 5: Fitting of the real part of the GPDF. Left: $Z^{(2)}(u)$, deuterium; right: $Z^{(0)}(u)$, hydrogen. 
mination of the fitting coefficients for both required 4.6". Clearly, the time required by the interface between the two codes is practically negligible.

We finally note that for a given numerical resolution, the TORIC execution time is strictly linear in the number of toroidal modes included. By parallelizing the fully independent solution of the wave equations for the different modes, a complete spectral scan could be executed almost as fast as simulations using a single representative mode. The execution time of SSFPQL increases quadratically with the number of Legendre polynomials used in eq. 8 , but is strictly linear in the number of magnetic surfaces on which the kinetic equation is solved. For very large problems, therefore, one could easily parallelize the solution at different points in the radial mesh.

\section{Conclusions.}

The interface implemented to transmit the relevant information about the quasilinear ion distribution functions from the solver of the kinetic equation SSFPQL to the solver of Maxwell's equations TORIC allows a considerable economy of memory and CPU time during execution of the iterative simulations of IC heating in tokamak plasmas, without significantly sacrificing accuracy. The efficiency of this interface allows one to perform convergent simulations of IC heating in present-day tokamaks on a personal computer, and makes the package TORIC-SSFPQL a useful tool also for simulations of larger plasmas such as JET and ITER. As an example of the possibilities offered by the package, we have been able to compare with a modest effort simulations using a single representative toroidal mode with the same simulations taking into account the full toroidal spectrum radiated by the antenna. While in the case of heating with symmetrically excited antennas the improvement in accuracy is modest, the full-spectre scan is a necessity with other excitations, particularly when current drive is attempted, as recently stressed by Dumont [17].

The main lines of our approach can in principle be extended to other packages. Its efficiency, however, is based in part on the fact that the kinetic equation solved by SSFPQL is surface averaged, so that it can be integrated independently on each magnetic surface. This is also the main limitation of our physical model. In the case of more sophisticated kinetic solvers based 
on orbit averaging and/or Monte Carlo methods, some modifications of the structure of this interface will be unavoidable.

\section{References}

[1] C. Gormezano, A. Sips, T. Luce, S. Ide, A. Becoulet, X. Litaudon, A. Isayama, J. Hobirk, M. Wade, T. Oikawa, R. Prater, A. Zvonkov, B. Lloyd, T. Suzuki, E. Barbato, P. Bonoli, C. Phillips, V. Vdovin, E. Joffrin, T. Casper, J. Ferron, D. Mazon, D. Moreau, R. Bundy, C. Kessel, A. Fukuyama, N. Hayashi, F. Imbeaux, M. Murakami, A. Polevoi, H. S. John, Nuclear Fusion 47 (2007) S285-S336.

[2] E. F. Jaeger, L. A. Berry, S. D. Ahern, R. F. Barrett, D. B. Batchelor, M. D. Carter, E. F. D'Azevedo, R. D. Moore, R. W. Harvey, J. R. Myra, D. A. D'Ippolito, R. J. Dumont, C. K. Phillips, H. Okuda, D. N. Smithe, P. T. Bonoli, J. C. Wright, M. Choi, Physics of Plasmas 13 (2006) 056101.

[3] S. Murakami, T. Yamamoto, F. A, J. Talmadge, K. Likin, J. Radder, Contrib. Plasma Phys. 50 (2010) 546.

[4] M. Brambilla, R. Bilato, Nuclear Fusion 49 (2009) 085004.

[5] R. Bilato, M. Brambilla, O. Maj, L. Horton, C. Maggi, J. Stober, Nuclear Fusion 51 (2011) 103034.

[6] M. Choi, D. Green, W. W. Heidbrink, R. Harvey, D. Liu, V. S. Chan, L. A. Berry, F. Jaeger, L. L. Lao, R. I. Pinsker, M. Podesta, D. N. Smithe, J. M. Park, P. Bonoli, R. SciDAC, S. Team, Physics of Plasmas 17 (2010) 056102.

[7] B. D. Fried, S. D. Conte, The plasma dispersion function, N.Y. : Academic Press, 1961.

[8] J. C. Wright, E. J. Valeo, C. K. Phillips, P. T. Bonoli, M. Brambilla, Communincations in Computational Physics 4 (2008) 545.

[9] W. Gautschi, SIAM J. Num. Anal. 7 (1970) 187.

[10] R. Dumont, in: RADIO FREQUENCY POWER IN PLASMAS: 18th Topical Conference on Radio Frequency Power in Plasmas, volume 1187, AIP, 2009, p. 97. 
[11] M. Brambilla, Plasma Physics and Controlled Fusion 41 (1999) 1-34.

[12] M. Brambilla, Physics Letters A 188 (1994) 376 - 383.

[13] E. J. Valeo, C. K. Phillips, P. T. Bonoli, J. C. Wright, M. B. R. S. Team, in: RADIO FREQUENCY POWER IN PLASMAS: 17th Topical Conference on Radio Frequency Power in Plasmas, volume 933, AIP, 2007, pp. 297-300.

[14] R. Bilato, M. Brambilla, Z. Jiang, Journal of Physics: Conference Series 401 (2012) 012001.

[15] A. Herrmann, O. Gruber, Fusion Science and Technology 44 (2003) 569.

[16] M. Brambilla, Nucl. Fusion 38 (1998) 1805.

[17] R. Dumont, D. Zarzoso, Nuclear Fusion 53 (2013) 013002. 